\title{
Suppurative appendicitis at colonoscopy
}

A 65-year-old man came to our emergency department with complaints of diffuse abdominal pain and a change in bowel habits for the past 4 days. He denied nausea, vomiting or fever. His medical history included diabetes mellitus, arterial hypertension, cerebral stroke, and ischemic heart disease. He had no past surgical history. On presentation, he was in good general condition and the abdomen was soft, depressive, with diffuse discomfort on palpation in the right upper quadrant, but without Blumberg sign. Laboratory tests showed leukocytosis $(11 \mid 700 / \mu \mathrm{L})$, hemoglobin at $14 \mathrm{~g} / \mathrm{dL}$, and C-reactive protein at $8.7 \mathrm{mg} / \mathrm{dL}$ (normal range $<1.0 \mathrm{mg} / \mathrm{dL}$ ). Abdominal radiography and ultrasonography were normal. He was admitted with suspicion of diverticulitis.

He was still complaining of pain 5 days later, despite analgesia medication. A colonoscopy was performed, which showed a suppurative appendicitis ( $\bullet$ Fig. 1).

After the procedure, surgical consultation was immediately requested and an abdominal computed tomography (CT) was performed. CT scan revealed an inflammatory mass with gas inside the right lower quadrant, without free peritoneal liquid. The patient underwent appendectomy and abscess drainage, and postoperative recovery was uneventful.

The diagnosis of acute appendicitis is based on clinical criteria, corroborated by laboratory data and image findings [1]. However, some situations have subtle or atypical presentations and the role of colonoscopy has been shown to be particularly useful in these situations [1 - 3].

\section{Competing interests: None}

Endoscopy_UCTN_Code_CCL_1AD_2AJ

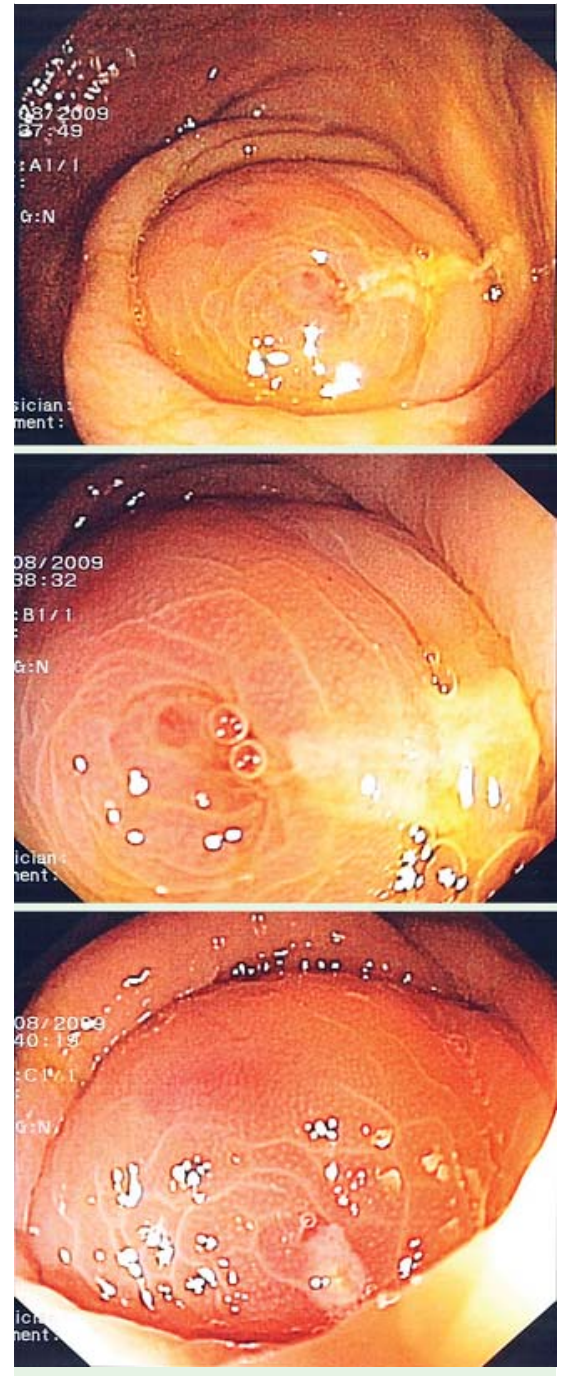

P. Duarte, R. Ramos, C. Casteleiro Alves Gastroenterology Department, Centro Hospitalar Cova da Beira, E.P.E., Covilhã, Portugal

\section{References}

1 Petro M, Minocha A. Asymptomatic early acute appendicitis initiated and diagnosed during colonoscopy: a case report. World J Gastroenterol 2005; 34: 5398-5400

2 Liu CH, Tsai FC, Hsu SJ, Yang PM. Successful colonoscopic drainage of appendiceal pus in acute appendicitis. Gastrointest Endosc 2006; 64: 1011-1012

3 Boix J, Lorenzo-Zúñiga $V$, Moreno de Vega $V$, Gassull MA. Acute appendicitis diagnosed with high-resolution endoscopy plus narrow-band imaging. Endoscopy 2006; 38: E45

Bibliography

DOI $10.1055 / \mathrm{s}-0029-1243908$

Endoscopy 2010; 42: E104

(c) Georg Thieme Verlag KG Stuttgart - New York . ISSN 0013-726X

\section{Corresponding author}

\section{P. Duarte, MD}

Gastroenterology Department

Centro Hospitalar Cova da Beira, E.P.E.

Quinta do Alvito

6200-251 Covilhã

Portugal

Fax: +351-275-330001

patriciaduartecovilha@iol.pt
Fig. 1 Appendix bulging into cecal lumen with purulent exudation through its orifice. 